\title{
Effect of alpha-lipoic acid on radiation-induced small intestine injury in mice
}

\author{
Bae Kwon J eong ${ }^{1,4}$, J in Ho Song ${ }^{1,4}$, Hojin J eong ${ }^{1,4}$, Hoon Sik Choi ${ }^{1}$, J ung Hwa J ung ${ }^{2,4}$, \\ Jong Ryeal Hahm ${ }^{2,4}$, Seung Hoon Woo ${ }^{3,4}$, Myeong Hee J ung ${ }^{5}$, Bong-Hoi Choi ${ }^{6}$, Jin \\ Hyun Kim ${ }^{4,5}$, Ki Mun Kang ${ }^{1,4}$ \\ ${ }^{1}$ Department of Radiation Oncology, Gyeongsang National University School of Medicine and Gyeongsang National University \\ Hospital, J inju, Republic of Korea \\ ${ }^{2}$ Department of Internal Medicine, Gyeongsang National University School of Medicine and Gyeongsang National University \\ Hospital, Jinju, Republic of Korea \\ ${ }^{3}$ Department of Otolaryngology, Gyeongsang National University School of Medicine and Gyeongsang National University Hospital, \\ Jinju, Republic of Korea \\ ${ }^{4}$ Institute of Health Science, Gyeongsang National University, Jinju, Republic of Korea \\ ${ }^{5}$ Biomedical Research Institute, Gyeongsang National University Hospital, Jinju, Republic of Korea \\ ${ }^{6}$ Department of Nuclear Medicine and Molecular Imaging, Gyeongsang National University, Jinju, Republic of Korea \\ Correspondence to: Ki Mun Kang, e-mail: jsk92@gnu.ac.kr \\ Jin Hyun Kim, e-mail: ajini7044@hanmail.net
}

Keywords: radiation therapy, alpha-lipoic acid, small intestine, oxidative stress, inflammation

Received: October 05, 2015

Accepted: January 23, 2016

Published: March 03, 2016

\section{ABSTRACT}

Purpose: Radiation therapy is a highly effective treatment for patients with solid tumors. However, it can cause damage and inflammation in normal tissues. Here, we investigated the effects of alpha-lipoic acid (ALA) as radioprotection agent for the small intestine in a mouse model.

Materials and Methods: Whole abdomen was evenly irradiated with total a dose of $15 \mathrm{~Gy}$. Mice were treated with either ALA ( $100 \mathrm{mg} / \mathrm{kg}$, intraperitoneal injection [i.p.]) or saline (equal volume, i.p.) the prior to radiation as $100 \mathrm{mg} / \mathrm{kg} / \mathrm{day}$ for 3 days. Body weight, food intake, histopathology, and biochemical parameters were evaluated.

Results: Significant differences in body weight and food intake were observed between the radiation (RT) and ALA + RT groups. Moreover, the number of crypt cells was higher in the ALA + RT group. Inflammation was decreased and recovery time was shortened in the ALA + RT group compared with the RT group. The levels of inflammation-related factors (i.e., phosphorylated nuclear factor kappa $B$ and matrix metalloproteinase-9) and mitogen-activated protein kinases were significantly decreased in the ALA + RT group compared with those in the RT group.

Conclusions: ALA treatment prior to radiation decreases the severity and duration of radiation-induced enteritis by reducing inflammation, oxidative stress, and cell death.

\section{INTRODUCTION}

Cancer is the leading cause of death in economically developed countries and the second leading cause of death in developing countries in 2011 [1]. Management of cancer is the major goal of oncology physicians and radiation therapy, alone or combined with chemotherapy or surgery, is the standard treatment modality for cancer. The aim of radiation therapy in the management of cancer is to maximize the effects of radiation therapy by increasing the radiation dose to the cancer and concurrently minimize side effects by decreasing the radiation dose to the normal organs, thereby increasing the therapeutic ratio [2]. It is important to improve the patient's quality of life, and survival can be prolonged through effective cancer management by using appropriate and effective radiation therapy.

Enteritis is the most common side effect of radiation therapy in the treatment of abdominal or pelvic cancer, including gastric, pancreatic, rectal, cervical, and endometrial cancers [3], and causes decreased quality of 
life through poor oral intake and absorption of nutrition. In the small intestine, the tolerance dose, defined as the highest dose of radiation that normal organs can endure, acts as a limiting factor for the radiation dose and is a predictive factor for radiation-induced enteritis. Radiationinduced enteritis is classified into six grades $(0-5)$ depending on its severity [4].

Several methods have been proposed to enhance the efficacy or reduce the side effects of radiation therapy. One method for enhancing the efficacy of radiation therapy is to combine radiation therapy with other treatment modalities, such as surgery or chemotherapy. The purpose of combined therapy is to increase the sensitivity of the tumor to radiation therapy $[3,5]$. Alternatively, the therapeutic ratio can be increased using advanced techniques such as image-guided radiation therapy or stereotactic radiosurgery, resulting in an increase in irradiation dose to the target volume without increase in irradiation dose to the normal organs [5]. Moreover, the side effects of radiation therapy can be reduced by conducting high technique of radiation therapy, as mentioned above, and by decreasing the irradiated volume of normal organs $[5,6]$. Another method to reduce side effects is to decrease the sensitivity of normal organs to radiation by using radioprotective agents rather than increasing the sensitivity of tumors by using radiation sensitizers. Although many studies have examined the radioprotective effects of various agents [7-10], none has been used widely in clinical settings. Additionally, the ability of such agents to reduce the side effects caused by radiation therapy and the radioprotective effects of the agent on cancer cells are unclear.

Reactive oxygen species (ROS) generated by radiation causes radiation-induced complications, and thus antioxidant compounds have been used to scavenge these free radicals [11-14]. N-acetylcysteine and alpha lipoic acid (ALA), glutathione (GSH)-elevating agents, are typical antioxidant compounds. Both of which are non-toxic within certain concentration ranges in humans $[15,16]$. ALA is a strong antioxidant with high reactivity to free radicals and facilitates generation of vitamin $C$ and $\mathrm{E}$ to elevate tissue levels of GSH. ALA has been shown to be effective in preventing pathological processes involving ROS, such as ischemia-reperfusion injury, diabetes, hypertension, radiation injury, and human immunodeficiency virus activation [17-21]. Moreover, ALA is known to protect hematopoietic tissue against radiation injury when increasing the median lethal dose (LD50) and dose-reduction factor in mice [22].

Here, we aimed to investigate whether ALA could reduce the occurrence of radiation-induced enteritis in a mouse model.

\section{RESULTS}

\section{ALA alleviated the clinical symptoms of radiation-induced small intestinal injury}

The clinical severity of radiation-induced small intestinal injury was monitored by measuring body weight and food intake daily. No clinically significant weight loss or reduced food intake was observed in the control and ALA groups. However, irradiation induced marked decreases in body weight and food intake 3-10 and 2-10 days after radiation, respectively. The ALA + radiation (RT) group showed a moderate degree of weight loss and improved food intake following irradiation as compared with the RT group (Figure 1), suggesting that ALA may reduce clinical symptoms in the small intestine of mice subjected to radiation.

\section{ALA protected against radiation-induced small intestinal mucosal injury}

The height of villi reflects the degree of overall mucosal damage. Thus, we evaluated histological changes by measuring the small intestinal villus height. The control and ALA groups exhibited tall, well-arranged mucosal epithelial cells. However, irradiation caused severe mucosal damage (Figure 2A). Villus height decreased significantly in the RT group compared with the control and this decrease was suppressed in ALA + RT group at all experimental time-points (Figure 2B). Thus, ALA prevented radiation-induced morphological damage in the small intestine.

\section{ALA decreased apoptosis in the small intestine}

Apoptosis is a major pathogenic feature of radiationinduced small intestinal mucosal injury, and the degree of apoptosis reflects the degree of mucositis [2]. The degree of apoptosis was assessed using terminal deoxynucleotidyl transferase dUTP nick-end labeling (TUNEL) assay. TUNEL-positive signals in the small intestine significantly increased in the RT group compared with the control and ALA groups at each time point (Figure 3A). Most positive signals were detected at the edge of villi in the small intestinal mucosa. However, mice in the ALA + RT group exhibited a significant decrease in radiation-induced TUNEL-positive cells compared with the RT group (Figure 3B). These data indicate that ALA protected the small intestine against radiation-induced apoptosis.

\section{ALA restored GSH levels and reduced oxidative stress following radiation}

Tissue GSH levels decreased significantly in the RT group at 3 and 7 days after radiation. In the ALA + RT group, a significant increase was noted in tissue 
GSH levels compared with the RT group at 3 and 7 days after radiation; however, this difference was not significant at 14 days after radiation (Figure 4A). Ionizing radiation enhances the production of ROS, thereby inducing oxidative damage, including lipid peroxidation. Malondialdehyde (MDA) is a representative marker of lipid peroxidation. Therefore, we examined MDA expression in the small intestine of mice in the four groups. MDA-positive signals were mainly detected at the edges of villi and in the muscularis externa and serosa (Figure 4B). Radiation significantly increased the MDA-positive signals in the small intestine. ALA administration significantly decreased MDA expression induced by radiation at days 3 and 7 after radiation but the decrease was not significant at day 14 , similar to that observed for tissue GSH levels (Figure 4C). Additionally,
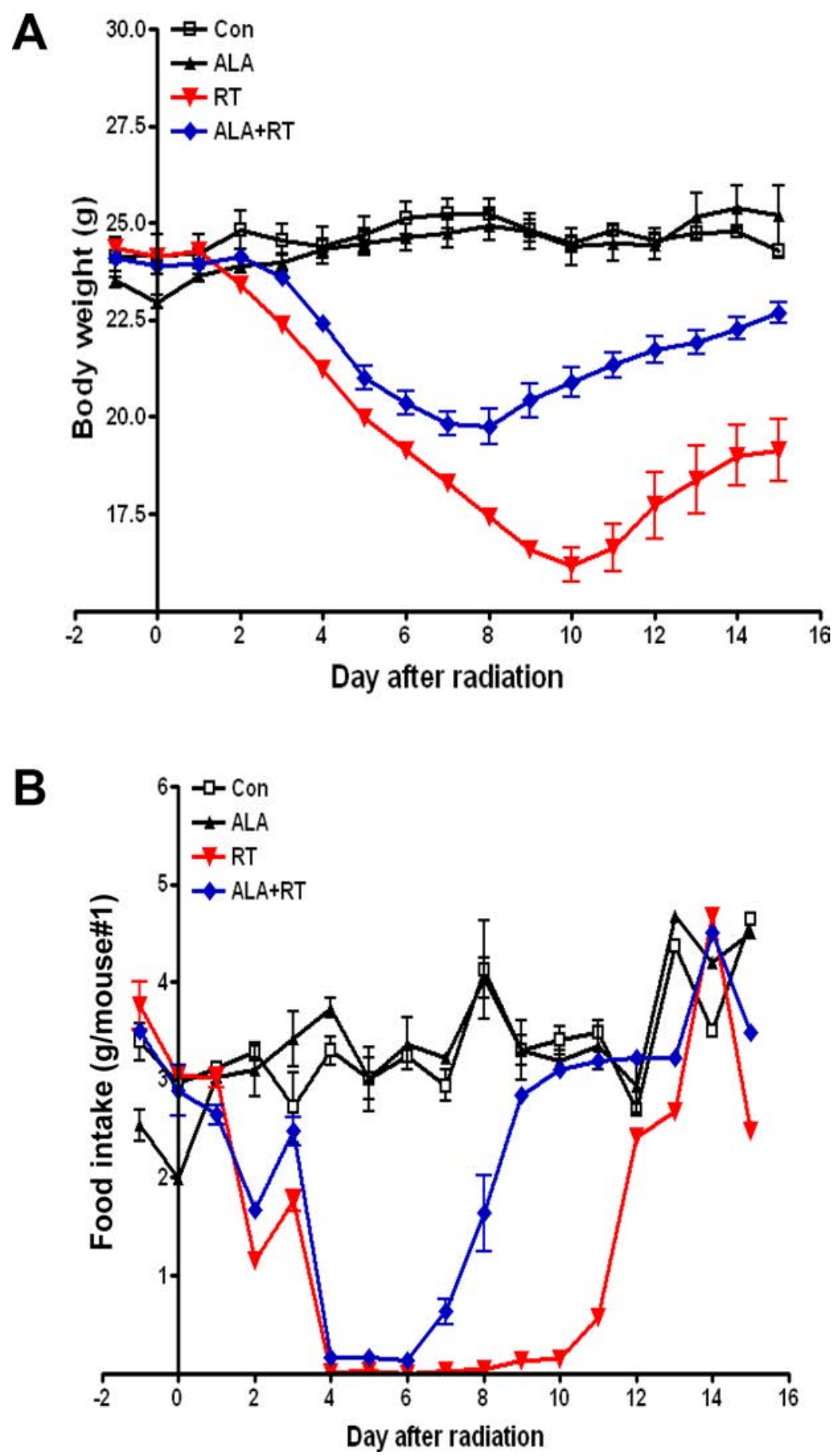

Figure 1: Changes in body weight and food intake in mice with radiation-induced small intestinal injury. Body weight and food intake were monitored daily in all mice. All experiments were repeated three times. Data are expressed as the mean \pm standard error (SE; $n=10$ mice/group). 
immunohistochemical staining of 8-hydroxy-2'deoxyguanosine (8-OHdG), a ROS-induced DNA damage marker, was performed to investigate the effects of ALA on radiation-induced oxidative stress (Figure 4D). 8-OHdGpositive cells were detected at the edges of the villi, significantly increased on day 3 and peaked on day 7 after

A

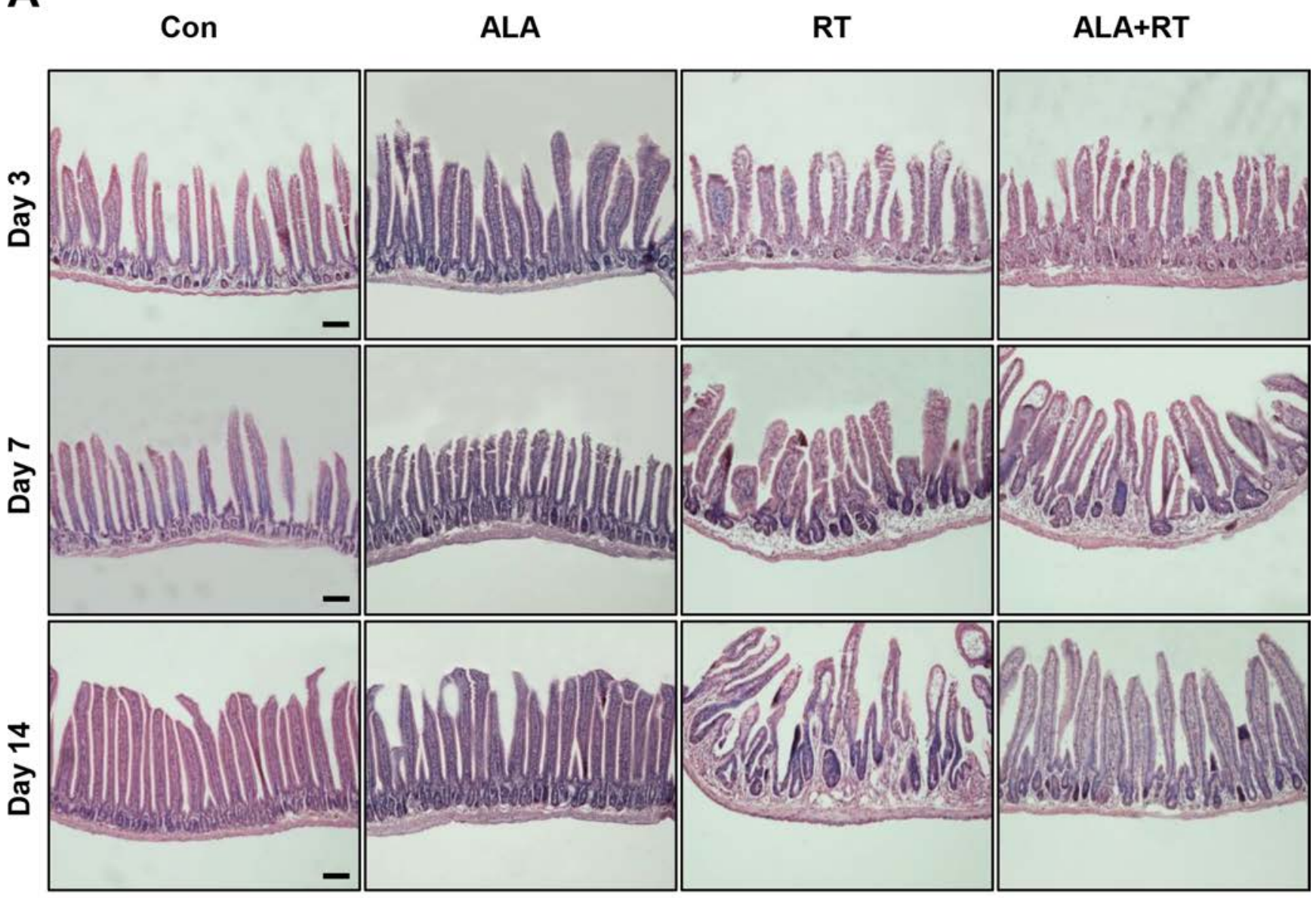

B

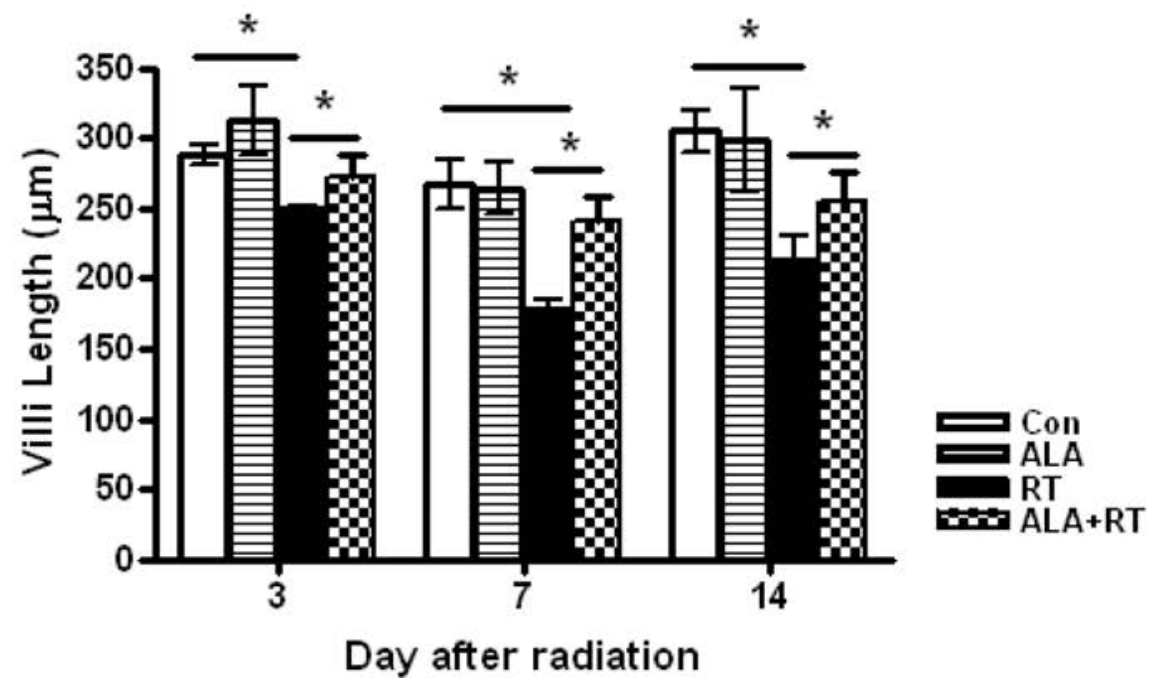

Figure 2: Histopathological changes of the small intestine at 3, 7, and 14 days after irradiation. Height measurements from 10 villi were obtained in small intestine sections from each group at $200 \times$ magnification. Each bar shows the mean $\pm \mathrm{SE} ;{ }^{*} p<0.05$ indicates differences between groups. Con: control group; RT: radiation group; ALA + RT: received alpha-lipoic acid (ALA) before irradiation. Scale bar; $100 \mu \mathrm{m}$. Con and ALA ( $n=4$ /each day), RT and ALA + RT ( $n=10 /$ each day). 


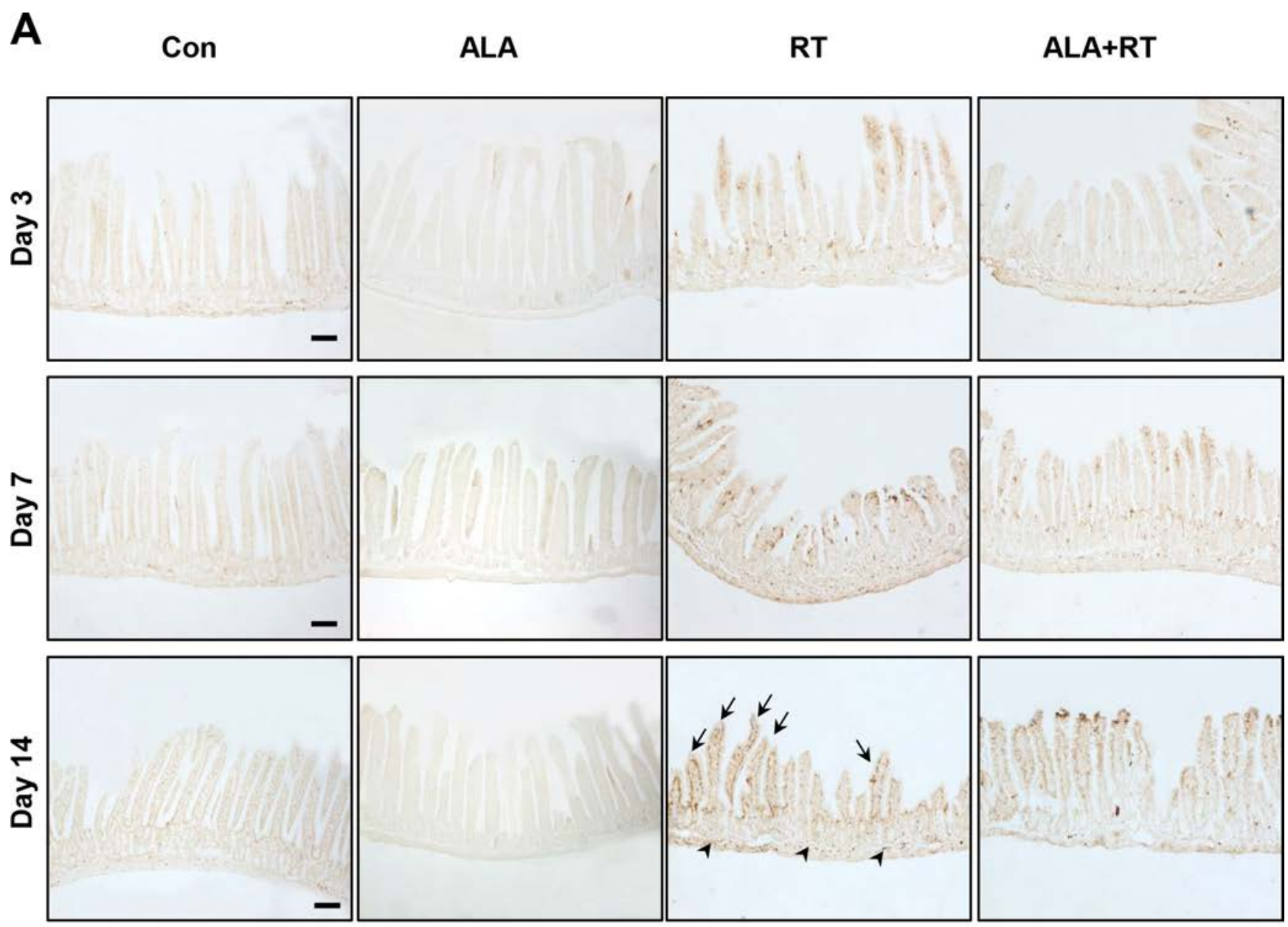

B

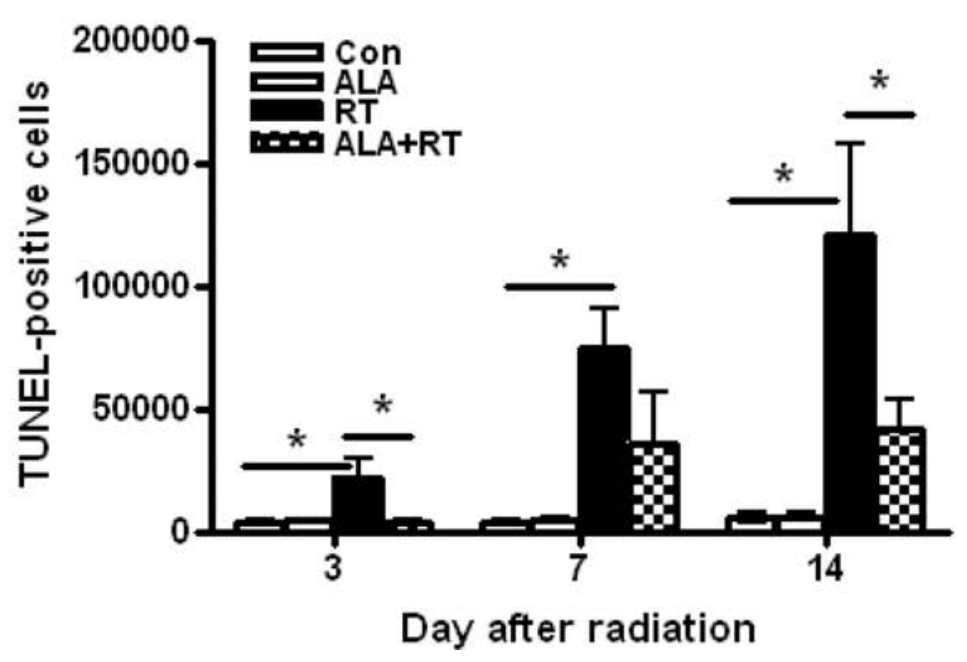

Figure 3: Apoptotic death in the small intestine with radiation-induced small intestinal injury. Apoptotic death was defined as the average number and density of terminal deoxynucleotidyl transferase dUTP nick-end labeling (TUNEL)-positive cells in 10 random fields from each section at 400× magnification (A). Signals density for TUNEL-positive cells was measured in the marked areas (arrow, edge of the villi. arrowhead, the muscularis mucosa). Each bar represents the mean \pm standard error (SE); ${ }^{*} p<0.05$ indicates differences between the radiation (RT) and alpha-lipoic acid (ALA) + RT groups (B). Con: control group; RT: radiation group; ALA + RT: received ALA before irradiation. Scale bar; $100 \mu \mathrm{m}$. Con and ALA ( $n=4 /$ each day), RT and ALA + RT ( $n=10 /$ each day). 
radiation, and these increases were remarkably suppressed in ALT + RT group (Figure 4E). Similar to the tissue GHS level and MDA expression, 8-OHdG expression did not significant alter at day 14 after radiation. These data suggest that ALA may reduce oxidative stress at the acute phase of radiation.

\section{ALA decreased MMP-9 expression, NF-кB phosphorylation, and serum IL-1 $\beta$ and IL-6 levels}

Radiation-induced enteritis is characterized by a defined pattern of inflammation and fibrosis, and MMP-9 is involved in the inflammatory response [23]. We found marked induction of MMP-9 expression in irradiated small intestine after 3, 7, and 14 days (Figure 5A). MMP-9 expression peaked on day 7, and ALA reduced MMP-9 expression at 3 and 7 days after radiation. The difference was not significant at 14 days after radiation (Figure 5B). In addition to MMP-9, the NF- $\kappa \mathrm{B}$ signaling pathway is another major source of inflammation in radiation-induced small intestinal injury [24]. Thus, we next evaluated the activation of NF- $\kappa$ B. Marked induction of phosphorylated $\mathrm{NF}-\kappa \mathrm{B}$ protein was observed in irradiated small intestinal tissues after 3, 7, and 14 days, peaking at day 7 after irradiation and with high levels maintained until day 14 (Figure 5A). ALA treatment reduced the phosphorylation of NF- $\kappa \mathrm{B}$ at all-time points (Figure $5 \mathrm{~B}$ ). We also found that IL-1 $\beta$ and IL-6, two main inflammatory cytokines, increased significantly in irradiated mice and the levels were ameliorated by ALA treatment on day 7 after radiation. In IL- $1 \beta$, the level is also significantly decreased by ALA on day 3 after radiation (Figure 5C). No significant differences found between control and ALA alone group in serum IL-1 $\beta$ and IL-6 (data not shown). No significant expression differences were observed for MMP-9 and NF- $\kappa \mathrm{B}$ in the control and ALA groups (Figure 6). These data suggest that ALA decreased inflammation in irradiated small intestinal tissues.

\section{DISCUSSION}

Radiation therapy is one of the primary modalities for treating patients with cancers of the pelvic cavity or abdomen [25-28]. Enteritis is the most common side effect of radiation therapy for the treatment of abdominal cancer, and the susceptibility of the small intestine to radiation-induced damage is the limiting factor for the prescription dose of radiation therapy. Enteritis caused by radiation therapy, which is accompanied by various clinical symptoms, reduces the patient's quality of life and increases the cost of treatment and the social health care cost [29, 30]. Currently, to our knowledge, there are no widely used methods for reducing the occurrence or severity of radiation-induced enteritis. We aimed to investigate the effect of ALA in the prevention or treatment of radiation-induced enteritis. Our results showed that administration of ALA prior to radiation significantly alleviated the clinical symptoms of radiation-induced enteritis, including reduced food intake and weight loss. These effects may be explained by reductions in cell death, oxidative stress, and inflammation.

Our results showed reductions in food intake and weight loss similar to those in patients with pelvic or abdominal cancer who underwent radiation therapy. These symptoms were less serious and recovered more rapidly in the ALA-treated group. The observed weight loss may have resulted from poor food intake caused by shortening of the villi after radiation. Tissue damage, which began in the submucosal area and migrated to the epithelium, was lower in the ALA + RT group than in the RT group. The height of small intestinal villi were greater in the

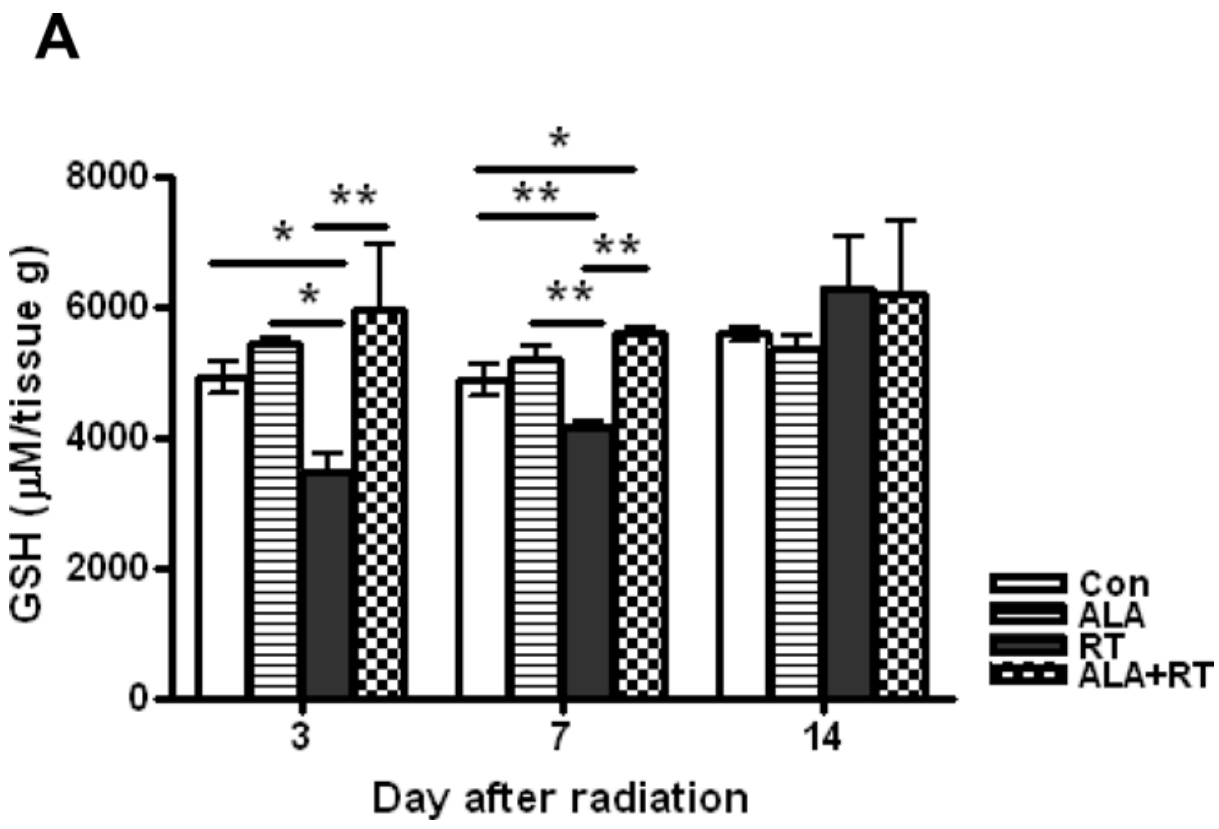



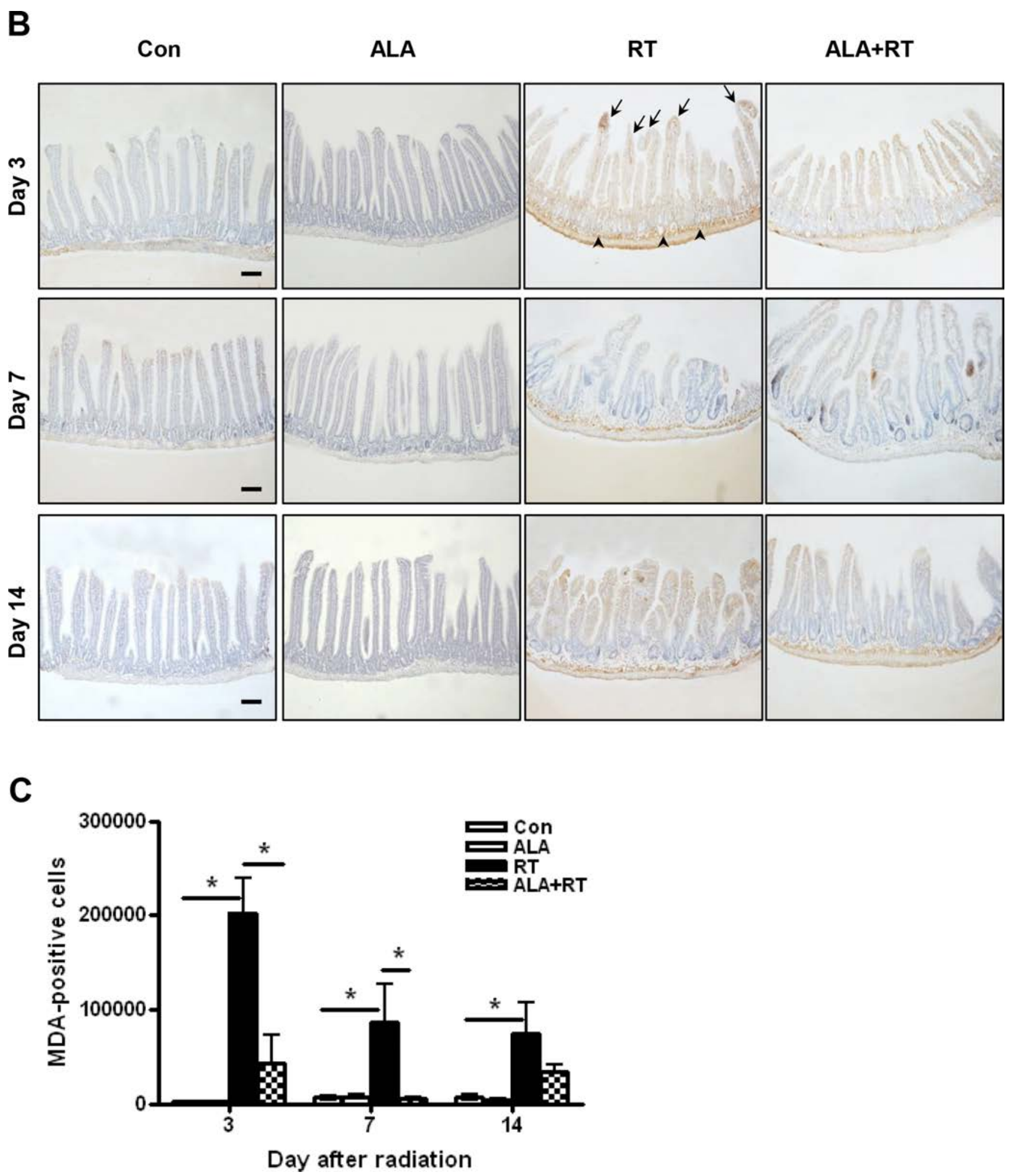
D

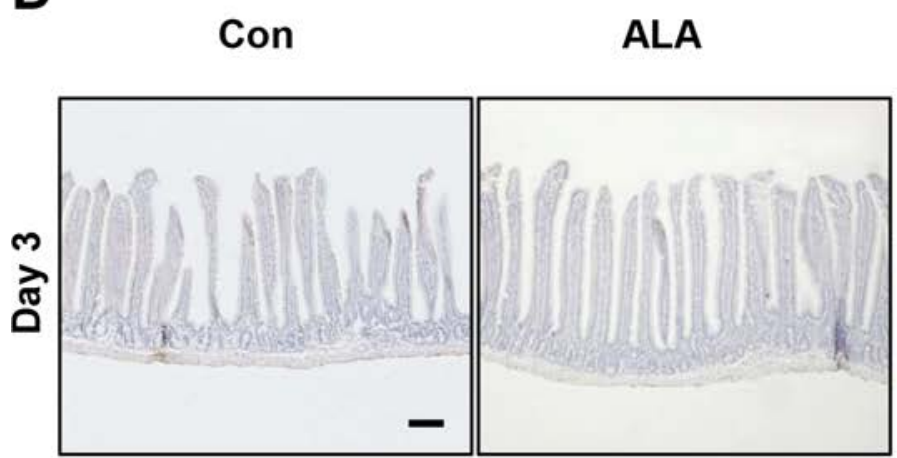

RT

ALA+RT
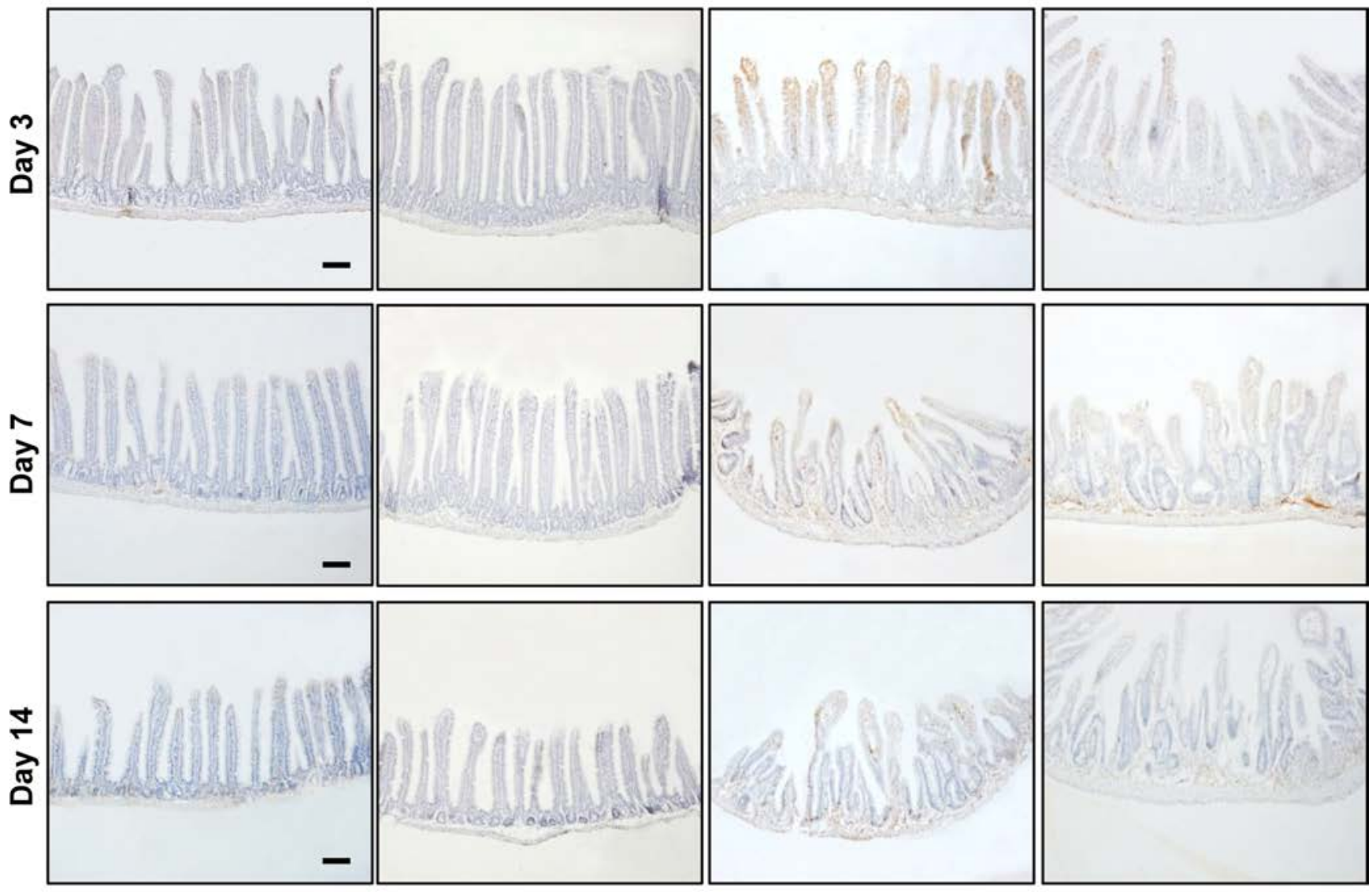

$\mathbf{E}$
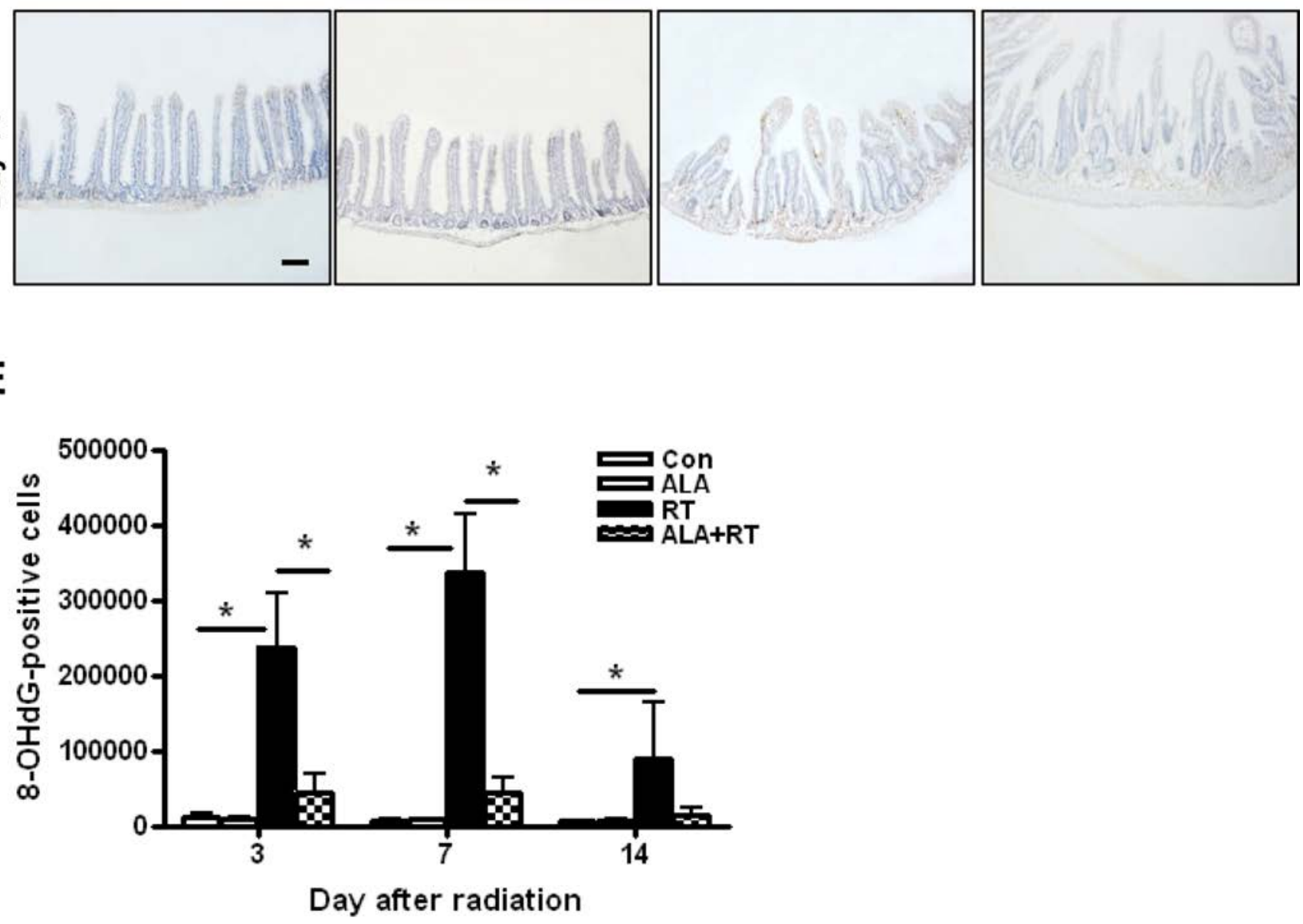

Figure 4: Oxidative stress induced by radiation. Small intestinal tissue glutathione (GSH) levels were measured. Data are the mean \pm standard error $(\mathrm{SE}) ;{ }^{*} p<0.05$ and ${ }^{*} p<<0.05$ indicate differences between groups (A). Malondialdehyde (MDA) was predominantly expressed at the edges of villi and in the muscularis externa and serosa (B). 8-Hydroxy-2'-deoxyguanosine (8-OHdG)-positive cells were localized at the small intestinal mucosa and submucosa and the muscularis externa and serosa (D). Signals density for MDA and 8-OHdGpositive cells were measured in the marked areas (arrow, edge of the villi. arrowhead, the muscularis mucosa). Data are the mean \pm standard error $(\mathrm{SE}){ }^{*} p<0.05$ indicate differences between groups (C and E). Scale bar; $100 \mu \mathrm{m}$. Con: control group; RT: radiation group; ALA + RT: received alpha-lipoic acid (ALA) before irradiation. Con and ALA ( $n=4 /$ each day), RT and ALA + RT ( $n=10 /$ each day). 


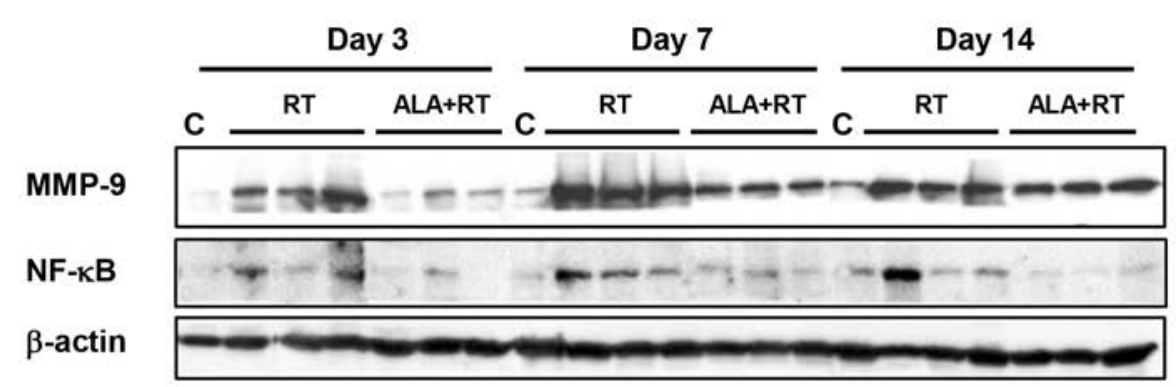

B
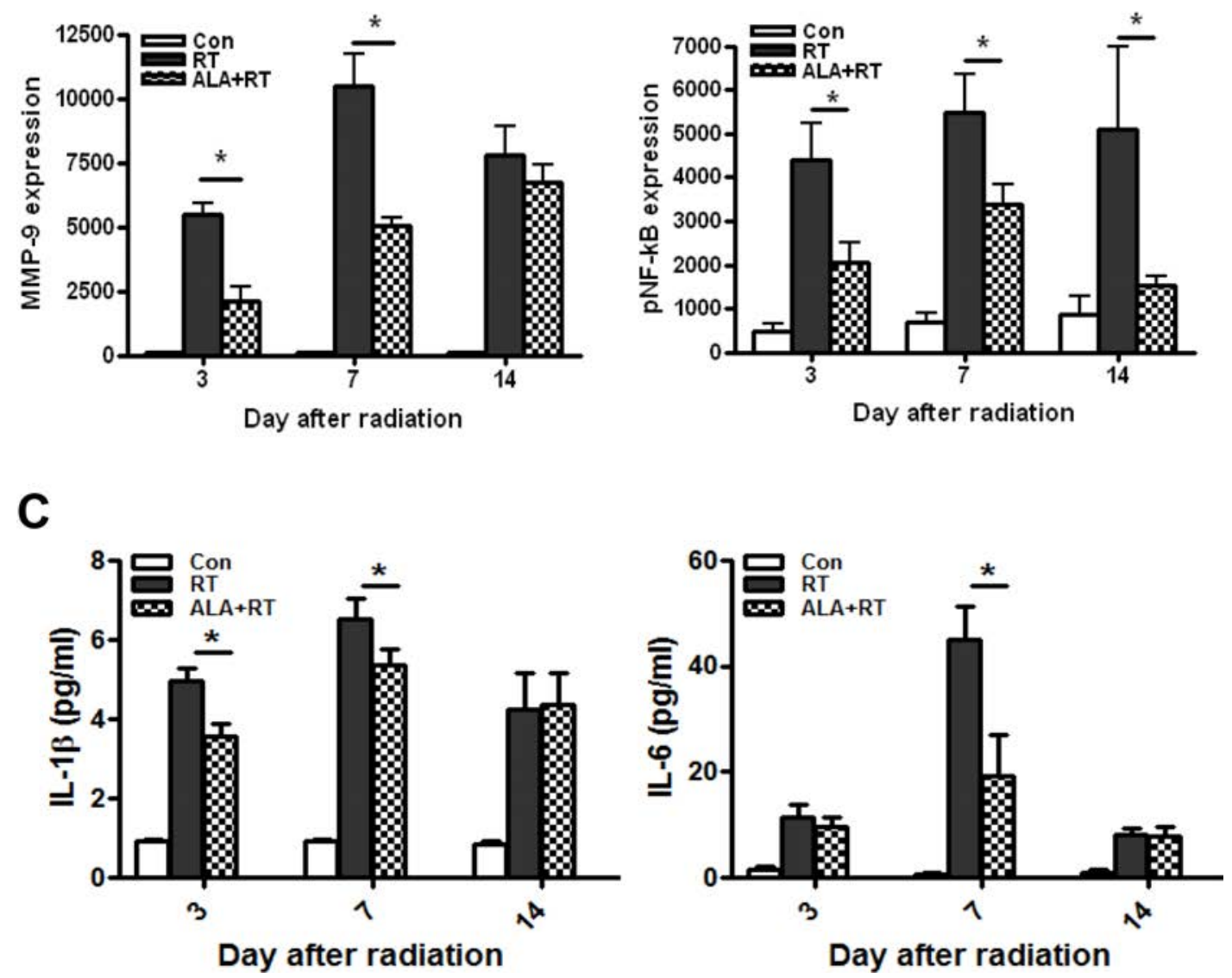

Figure 5: Western blotting was performed using anti-MMP-9 and anti-phosphorylated NF- $\mathrm{B}$ antibodies. $\beta$-actin was evaluated as the loading control (A). Alpha-lipoic acid (ALA) inhibited radiation-induced expression of MMP-9 and phosphorylated of NF- $\mathrm{BB}$ in the RT-induced small intestine. Con ( $n=3$ /each day), RT and ALA + RT ( $n=9 /$ each day, $n=3 /$ each lane) (B). ELISA analysis of serum IL-1 $\beta$ and IL-6 levels from each group on each day. Con $(n=5 /$ each day), RT and ALA + RT ( $n=10 /$ each day and group) (C). Data are the mean \pm standard error (SE); ${ }^{*} p<0.05$ indicate differences between groups. Con: control group; RT: radiation group; ALA + RT: received ALA before irradiation.

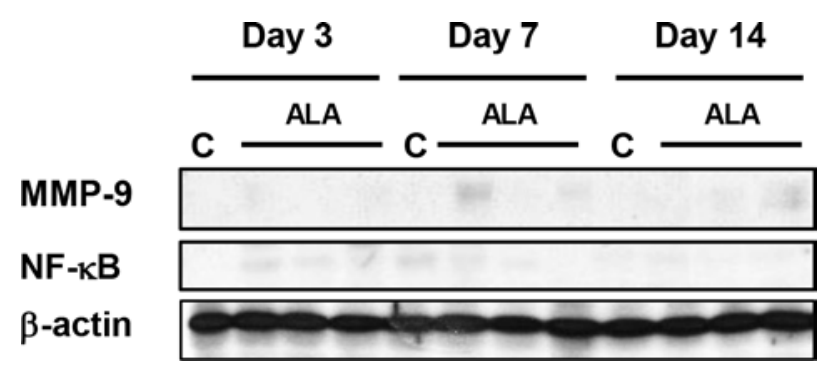

Figure 6: Western blotting was performed using anti-MMP-9 and anti-phosphorylated NF-кB antibodies. $\beta$-actin was evaluated as the loading control. Con: control group, ALA: alpha-lipoic acid (ALA) alone group. Con and ALA ( $n=3 /$ each day). 
Table 1: Experimental design and time table for treatment

\begin{tabular}{|l|c|c|c|c|c|c|c|c|c|c|c|c|c|c|c|c|c|}
\hline & 2 & 1 & 0 & 1 & 2 & 3 & 4 & 5 & 6 & 7 & 8 & 9 & 10 & 11 & 12 & 13 & 14 \\
\hline ALA & $\sqrt{ }$ & $\sqrt{ }$ & $\sqrt{ }$ & & & & & & & & & & & & & & \\
\hline Radiation & & & $\sqrt{ }$ & & & & & & & & & & & & & & \\
\hline Sampling & & & & & & $\sqrt{ }$ & & & & $\sqrt{ }$ & & & & & & & $\sqrt{ }$ \\
\hline
\end{tabular}

Thirty-nine BALB/c mice were randomized to four groups: i) control group (9 mice), ii) ALA group (9 mice), iii) RT group (11 mice), and RT + ALA group (10 mice). Small intestine damage was induced by irradiation (15 Gy, 1 fraction) on the abdomen. In the ALA group, mice received $100 \mathrm{mg} / \mathrm{kg} /$ day ALA by intraperitoneal injection on days -2 to 0 . The control group received vehicle in the same way. The small intestine was collected 3rd, 7th, and 14th days after irradiation.

$\mathrm{ALA}+\mathrm{RT}$ group than in the RT group. Thus, these data showed that ALA could attenuate radiation-induced bowel damage. The migration of tissue damage was in line with the traditional concept that radiation-induced tissue damage is more rapid and severe in rapidly proliferating tissue [2].

The GSH level was measured to verify the role of ALA in oxidative stress, which disrupts DNA damage and normal cellular signaling and is involved in various clinical diseases, particularly cancer [31-33]. The observed temporal changes in GSH levels in the RT group supported a normal response to radiation. GSH levels were significantly lower in the RT group than in the ALA + RT group, because antioxidants were consumed to reduce radiation-induced oxidative stress. GSH levels increased by internal homeostasis mechanisms on day 14 after irradiation, although no differences were noted between the RT and ALA + RT groups.

The mechanism by which radiation eliminates cancer cells involves the production of free radicals inside the body and destruction of the DNA structure of cancer cells, leading to cell death [2]. However, in clinical practice, this phenomenon occurs not only in cancer cells but also in normal tissues affected by radiation. Preventing DNA damage and subsequent cell death in the normal tissues can reduce the side effects of radiation. To determine the potential differences in level of cell death caused by ALA, we used 8-OHdG as a marker of DNA damage induced by ROS [34, 35], and performed TUNEL assay to estimate cellular apoptosis [36]. 8-OHdG staining showed that DNA damage was inhibited by ALA, consistent with the observed reduced morphological damage in the ALA + RT group. Moreover, we also observed marked differences in apoptosis between the RT and RT + ALA groups. The tendency for increased apoptosis in the RT group may have been caused by mitotic catastrophe, in which apoptosis occurred in cells with DNA damage during the following meiosis. The changes in the localization of the signal over time for both $8-\mathrm{OHdG}$ and TUNEL staining suggest migration from the submucosa to epithelium, as supported by hematoxylin and eosin (H \& E) staining. Radiation-induced cell membrane damage, as measured by MDA staining [37], also reduced due to ALA. These results suggest that pretreatment with ALA before radiation could reduce cellular DNA damage or membrane damage induced by free radicals and ultimately reduce the side effects of radiation by preventing cell death in normal tissues.

Inflammation that occurs after irradiation can be induced by a series of processes, including ROS, DNA damage, lipid peroxidation, and apoptosis. Although inflammation is necessary for resolving various types of damage in the body, inflammation occurring after radiation causes various clinical symptoms, and continuous inflammation may result in severe consequences such as cancer. Therefore, resolving inflammation as early is generally beneficial. We evaluated the activation of NF- $\kappa$ B, which plays a crucial role in the immune response against inflammation induced by various stimuli [24], and the expression of MMP-9 [23], which is regulated by NF- $\mathrm{kB}$ and is involved in inflammation, tissue repair, and tissue remodeling. Although the pattern of MMP- 9 expression in response to radiation was similar to that of NF- $\mathrm{kB}$ activation over time, MMP-9 expression in the ALA + RT group tended to increase continuously with time, suggesting that MMP-9 is involved in tissue remodeling, which is associated with fibrosis, a long-term side effect of radiation subsequent to inflammation.

Here, the changes in the small intestine induced by radiation may have been caused by ROS-dependent oxidative stress, triggering DNA and tissue damage and inflammation. It is likely that the apoptosis induced by DNA damage leads to cell death. Inflammation would then occur continuously through factors involved in tissue damage or recovery. In this presumed series of processes, we found that ALA reduced tissue damage, apoptosis, oxidative stress, and inflammation, and as a result, positive outcomes were observed in terms of changes in body weight and food intake.

Although we describe the protective effect of ALA against radiation in the current study, further studies are required to determine the mechanism underlying the effect of ALA. Moreover, we have not yet determined whether the protective effect of ALA against radiation also occurs in cancer cells targeted by radiation. To establish clinical applicability, it will be necessary to determine the differential effects of ALA in normal tissues and cancer cells. 
In conclusion, ALA treatment prior to radiation decreased the severity and duration of radiation-induced enteritis by reducing tissue damage, cell death, oxidative stress, and inflammation. Thus, ALA has radioprotective effects against radiation-induced enteritis.

\section{MATERIALS AND METHODS}

\section{Animals}

Male BALB/c mice (7-8 weeks age) were obtained from Koatech Animal Inc. (Peongtaek, Korea). The mice were housed under specific pathogen-free conditions at four animals per polycarbonate cage, with free access to standard hamster food and water, in a room maintained at $23 \pm 1^{\circ} \mathrm{C}$ and $55 \pm 5 \%$ relative humidity, and a $12 \mathrm{~h}$ light/dark cycle (150-300 l×, with lights on from 06:00 to 18:00). All experiments were performed in compliance with institutional guidelines set by the Institutional Animal Care and Use Committee at the Gyeongsang National University (GLA-130621-M0039).

\section{Experimental design}

Forty BALB/c mice were randomized to four groups of 10 mice each, namely, the normal control ALA, RT, and RT + ALA groups. Small intestine damage was induced by irradiation to the abdomen. ALA was administered by intraperitoneal injection (i.p.) three times $(100 \mathrm{mg} / \mathrm{kg} / \mathrm{day}$, days -2 to 0$)$. Mice in the vehicle group received normal saline administered in the same manner. Blood samples were obtained via direct ventricular puncture, and the small intestine was collected at 3, 7, and 14 days after abdominal irradiation. Experiments were performed three times, independently.

\section{Radiation exposure}

Mice were anesthetized to immobilize them prior to radiation exposure. The whole abdomen was evenly irradiated with $2 \mathrm{~Gy} / \mathrm{min}$ (total dose of $15 \mathrm{~Gy}$ ) by using a photon 6-MV linear accelerator (21EX, Varian, Palo Alto, CA, USA). A 1.0-cm-tissue equivalent bolus was positioned on the abdomen to provide adequate buildup. Each mouse was exposed to a single dose of radiation.

\section{Histopathology}

Tissues were fixed in $4 \%$ paraformaldehyde in 0.1 M PBS, embedded in paraffin, and cut into 5- $\mu \mathrm{m}$-thick sections. The sections were stained with $\mathrm{H} \& \mathrm{E}$.

\section{Small intestinal histology: height of villi}

Segments of the ileum were collected, and the villus height (from the top of the villus to the villuscrypt junction) was measured by light microscopy using a calibrated micrometer $(200 \times)$. Ten intact and welloriented villi and crypts were measured and averaged for each sample.

\section{TUNEL assay}

The degree of apoptosis was assessed using TUNEL assay. DNA fragmentation was detected using a kit from Roche Applied Sciences (Indianapolis, IN, USA). Semiquantitative analysis was performed by counting the TUNEL-positive cells per field in the small intestinal tissue at $400 \times$ magnification. At least 10 areas in the cortex per slide were selected randomly. The mean number of brown cells in these selected fields was considered to be the number of TUNEL-positive cells. TUNEL-positive signals were analyzed by a blinded observer using NIS Elements BR3.1 (Nikon, Japan) software in 10 randomly selected fields.

\section{Measurement of GSH levels}

The GSH content in the small intestine was measured using Glutathione Assay kits (Sigma, St. Louis, MO, USA) according to the manufacturer's instructions. Briefly, $100 \mathrm{mg}$ of each tissue was homogenized in $0.5 \mathrm{~mL}$ of $\mathrm{GSH}$ reaction buffer containing $0.1 \mathrm{~mL}$ of $5 \%$ sulfosalicylic acid. To generate NADPH, $20 \mu \mathrm{L}$ of NADPH Generation Mix and $140 \mu \mathrm{L}$ of GSH reaction buffer were mixed and incubated at room temperature for $10 \mathrm{~min}$. Then, $20 \mu \mathrm{L}$ of either the GSH standard solution or the sample solution was added, followed by incubation at room temperature for 5-10 min. An additional $20 \mu \mathrm{L}$ of substrate solution was then added. A microplate reader was used to measure the absorbance at $405 \mathrm{~nm}$ (Molecular Devices Corp., Sunnyvale, CA, USA).

\section{Immunohistochemistry}

Immunohistochemical analysis was performed on 5- $\mu$ m-thick sections of paraformaldehyde-fixed, paraffin-embedded tissue using a Vectastain ABC kit (Vector Laboratories, CA, USA). Sections were blocked with $1 \%$ normal goat serum and then treated with antirabbit MDA (1:100 dilution, Santa Cruz Biotechnology, Santa Cruz, CA, USA) and anti-rat 8-OHdG (1:100 dilution, eBioscience, San Diego, CA, USA) antibodies at $4^{\circ} \mathrm{C}$ overnight in a humidified chamber. PBS-washed tissue sections were then incubated for $90 \mathrm{~min}$ at room temperature with secondary antibodies. Finally, the sections were incubated with avidin-biotinylated horseradish peroxidase (HRP)-complexes for $60 \mathrm{~min}$ at room temperature, rinsed in PBS, and developed using DAB with hydrogen peroxidase. The density and number of MDA- and 8-OHdG-positive signals were analyzed by a blinded observer by using NIS Elements BR3.1 (Nikon, Japan) software in 10 randomly selected fields. 


\section{Immunoblot}

Tissues were homogenized in lysis buffer. Proteins $(50 \mu \mathrm{g})$ were loaded on a sodium dodecyl sulfate-polyacrylamide gel. The blots were probed with polyclonal anti-matrix metalloproteinase (MMP)-9 (Cell Signaling Technology), and anti-phospho-nuclear factor kappaB (NF-kB; Santa Cruz Biotechnology) antibodies at $4^{\circ} \mathrm{C}$ overnight. Primary antibodies were visualized using secondary antibodies with an ECL kit (Amersham Pharmacia Biotech, Piscataway, NJ, USA).

\section{Measurement of serum IL-1 $\beta$ and IL-6}

Serum levels of IL-1 $\beta$ and IL-6 were quantified using specific enzyme-linked immunosorbent assay kits according to the manufacturer's instructions (R \& D system, Minneapolis, MN, USA).

\section{Statistical analysis}

Statistical analysis was conducted using Sigma Plot 7.0 software (SPSS Inc., Chicago, IL, USA). Results are presented as the means \pm standard errors of the mean. Differences between groups were assessed using one-way analysis of variance, followed by the Tukey test. $P \leq 0.05$ was considered significant.

\section{ACKNOWLEDGMENTS AND FUNDING}

This research was supported by the Gyeongsang National University Hospital (GNUHBIF-2014-0010) and Gyeongsang National University (RPP-2012-013). The founders had no role in the study design, data collection and analysis, decision to publish, or preparation of the manuscript.

\section{CONFLICTS OF INTEREST}

The authors declare no conflict of interest. The founding sponsors had no role in the design of the study; collection, analyses, or interpretation of data; writing of the manuscript; or in deciding to publish the results.

\section{REFERENCES}

1. Jemal A, Bray F, Center MM, Ferlay J, Ward E, Forman D. Global cancer statistics. CA Cancer J Clin. 2011; 61:69-90.

2. Hall EJ, Giaccia AJ. Radiobiology for the radiologist. 7th ed. Philadelphia: Wolters Kluwer Health/Lippincott Williams \& Wilkins; 2012.

3. Kavanagh BD, Pan CC, Dawson LA, Das SK, Li XA, Ten Haken RK, Miften M. Radiation dose-volume effects in the stomach and small bowel. Int J Radiat Oncol Biol Phys. 2010; 76:S101-107.
4. Sonis ST. The pathobiology of mucositis. Nat Rev Cancer. 2004; 4:277-284.

5. Pollom EL, Deng L, Pai RK, Brown JM, Giaccia A, Loo BW, Jr., Shultz DB, Le QT, Koong AC, Chang DT. Gastrointestinal toxicities tith combined antiangiogenic and stereotactic body radiation therapy. Int J Radiat Oncol Biol Phys. 2015; 92:568-576.

6. Dionisi F, Avery S, Lukens JN, Ding X, Kralik J, Kirk M, Roses RE, Amichetti M, Metz JM, Plastaras JP. Proton therapy in adjuvant treatment of gastric cancer: planning comparison with advanced x-ray therapy and feasibility report. Acta Oncol. 2014; 53:1312-1320.

7. Tannehill SP, Mehta MP. Amifostine and radiation therapy: past, present, and future. Semin Oncol. 1996;23:69-77.

8. Spencer CM, Goa KL. Amifostine. A review of its pharmacodynamic and pharmacokinetic properties, and therapeutic potential as a radioprotector and cytotoxic chemoprotector. Drugs. 1995; 50:1001-1031.

9. Oliai C, Yang LX. Radioprotectants to reduce the risk of radiation-induced carcinogenesis. Int J Radiat Biol. 2014; 90:203-213.

10. Rotovnik Kozjek N, Kompan L, Soeters P, Oblak I, Mlakar Mastnak D, Mozina B, Zadnik V, Anderluh F, Velenik V. Oral glutamine supplementation during preoperative radiochemotherapy in patients with rectal cancer: a randomised double blinded, placebo controlled pilot study. Clin Nutr. 2011; 30:567-570.

11. de Freitas Cuba L, Salum FG, Cherubini K, de Figueiredo MA. Antioxidant agents: a future alternative approach in the prevention and treatment of radiation-induced oral mucositis? Altern Ther Health Med. 2015; 21:36-41.

12. Olaku OO, Ojukwu MO, Zia FZ, White JD. The role of grape seed extract in the treatment of chemo/radiotherapy induced toxicity: A systematic review of preclinical studies. Nutr Cancer. 2015:1-11.

13. Zhao X, Yang H, Jiang G, Ni M, Deng Y, Cai J, Li Z, Shen F, Tao X. Simvastatin attenuates radiation-induced tissue damage in mice. J Radiat Res. 2014; 55:257-264.

14. Manda K, Ueno M, Moritake T, Anzai K. alpha-Lipoic acid attenuates $\mathrm{x}$-irradiation-induced oxidative stress in mice. Cell Biol Toxicol. 2007; 23:129-137.

15. Neal R, Matthews RH, Lutz P, Ercal N. Antioxidant role of $\mathrm{N}$-acetyl cysteine isomers following high dose irradiation. Free Radic Biol Med. 2003; 34:689-695.

16. Cremer DR, Rabeler R, Roberts A, Lynch B. Safety evaluation of alpha-lipoic acid (ALA). Regul Toxicol Pharmacol. 2006; 46:29-41.

17. Singh U, Jialal I. Alpha-lipoic acid supplementation and diabetes. Nutr Rev. 2008; 66:646-657.

18. Ghibu S, Richard C, Vergely C, Zeller M, Cottin Y, Rochette L. Antioxidant properties of an endogenous thiol: Alpha-lipoic acid, useful in the prevention of cardiovascular diseases. J Cardiovasc Pharmacol. 2009; 54:391-398. 
19. Rochette L, Ghibu S, Richard C, Zeller M, Cottin Y, Vergely C. Direct and indirect antioxidant properties of alpha-lipoic acid and therapeutic potential. Mol Nutr Food Res. 2013; 57:114-125.

20. Goraca A, Huk-Kolega H, Piechota A, Kleniewska P, Ciejka E, Skibska B. Lipoic acid - biological activity and therapeutic potential. Pharmacol Rep. 2011;63:849-858.

21. Koufaki M. Therapeutic applications of lipoic acid: a patent review (2011-2014). Expert Opin Ther Pat. 2014; 24:993-1005.

22. Ramakrishnan N, Wolfe WW, Catravas GN. Radioprotection of hematopoietic tissues in mice by lipoic acid. Radiat Res. 1992; 130:360-365.

23. Corbel M, Belleguic C, Boichot E, Lagente V. Involvement of gelatinases (MMP-2 and MMP-9) in the development of airway inflammation and pulmonary fibrosis. Cell Biol Toxicol. 2002; 18:51-61.

24. Morgan MJ, Liu ZG. Crosstalk of reactive oxygen species and NF-kappaB signaling. Cell Res. 2011; 21:103-115.

25. Suh WW, Blackstock AW, Herman J, Konski AA, Mohiuddin M, Poggi MM, Regine WF, Cosman BC, Saltz L, Johnstone PA. ACR Appropriateness Criteria on resectable rectal cancer: expert panel on radiation oncologyrectal/anal cancer. Int J Radiat Oncol Biol Phys. 2008; 70:1427-1430.

26. Goodman KA, Milgrom SA, Herman JM, Abdel-Wahab M, Azad N, Blackstock AW, Das P, Hong TS, Jabbour SK, Jones WE, 3rd, Konski AA, Koong AC, Kumar R, et al. ACR Appropriateness Criteria( $\mathrm{R})$ rectal cancer: metastatic disease at presentation. Oncology. 2014; 28:867-871, 876, 878.

27. Jones WE, 3rd, Thomas CR, Jr., Herman JM, AbdelWahab M, Azad N, Blackstock W, Das P, Goodman KA, Hong TS, Jabbour SK, Konski AA, Koong AC, et al. ACR appropriateness criteria( $\mathrm{R})$ resectable rectal cancer. Radiat Oncol. 2012; 7:161.
28. Konski AA, Suh WW, Herman JM, Blackstock AW, Jr., Hong TS, Poggi MM, Rodriguez-Bigas M, Small W, Jr., Thomas CR, Jr., Zook J. ACR Appropriateness Criteria(R)Recurrent Rectal Cancer. Gastrointest Cancer Res. 2012; 5:3-12.

29. Fyles AW, Dembo AJ, Bush RS, Levin W, Manchul LA, Pringle JF, Rawlings GA, Sturgeon JF, Thomas GM, Simm J. Analysis of complications in patients treated with abdomino-pelvic radiation therapy for ovarian carcinoma. Int J Radiat Oncol Biol Phys. 1992; 22:847-851.

30. Abayomi J, Kirwan J, Hackett A. The prevalence of chronic radiation enteritis following radiotherapy for cervical or endometrial cancer and its impact on quality of life. Eur J Oncol Nurs. 2009; 13:262-267.

31. Lennon SV, Martin SJ, Cotter TG. Dose-dependent induction of apoptosis in human tumour cell lines by widely diverging stimuli. Cell Prolif. 1991; 24:203-214.

32. Valko M, Morris H, Cronin MT. Metals, toxicity and oxidative stress. Curr Med Chem. 2005; 12:1161-1208.

33. Halliwell B. Oxidative stress and cancer: have we moved forward? Biochem J. 2007; 401:1-11.

34. Valavanidis A, Vlachogianni T, Fiotakis C. 8-hydroxy2'-deoxyguanosine (8-OHdG): A critical biomarker of oxidative stress and carcinogenesis. J Environ Sci Health C Environ Carcinog Ecotoxicol Rev. 2009; 27:120-139.

35. Wu LL, Chiou CC, Chang PY, Wu JT. Urinary 8-OHdG: a marker of oxidative stress to DNA and a risk factor for cancer, atherosclerosis and diabetics. Clin Chim Acta. 2004; 339:1-9.

36. Gavrieli Y, Sherman Y, Ben-Sasson SA. Identification of programmed cell death in situ via specific labeling of nuclear DNA fragmentation. J Cell Biol. 1992; 119:493-501.

37. Gawel S, Wardas $M$, Niedworok E, Wardas $P$. Malondialdehyde (MDA) as a lipid peroxidation marker. Wiad Lek. 2004; 57:453-455. 